\title{
Predicted Area Under the Curve Infinity by Dose
}

National Cancer Institute

\section{Source}

National Cancer Institute. Predicted Area Under the Curve Infinity by Dose. NCI

Thesaurus. Code C85786.

The area under the curve (AUC) extrapolated to infinity from dosing time, based on the predicted last concentration, divided by the dose. 